\title{
How ischaemic region shape affects ST potentials in models of cardiac tissue
}

\author{
J.P. Barnes ${ }^{\mathrm{a}, \mathrm{b}, *}$, P.R. Johnston ${ }^{\mathrm{a}, \mathrm{b}}$ \\ ${ }^{a}$ Queensland Micro- and Nanotechnology Centre, Griffith University, Brisbane, Queensland 4111, Australia \\ ${ }^{b}$ School of Biomolecular and Physical Sciences, Griffith University, Brisbane, Queensland 4111, Australia
}

\begin{abstract}
This numerical study uses a simple bidomain model of cardiac tissue to compare the effect of three different ischaemic region geometries (rectangular, cylindrical and semi-ellipsoidal) on the extracellular epicardial potentials during the ST segment. Results are obtained using anisotropic conductivities based on experimentally derived data. The model is then altered, to include heterogeneous conductivities in the ischaemic region and larger border zone widths, in order to better reproduce realistic scenarios. Initial results for the rectangular and cylindrical ischaemic shapes show a central depression over the ischaemic region, for low ischaemic thicknesses, which separates into three depressions as the ischaemic thickness increases. For ischaemic thicknesses above $70 \%$ an elevation appears over the ischaemic region and this increases in magnitude as the ischaemia becomes transmural. Results for the semi-ellipsoidal shape, however, differ, with the central depression separating into only two depressions as the thickness increases. Changing the conductivity inside the ischaemic region significantly affects results for each geometry, with depression staying over the ischaemic region for much higher levels of ischaemia (up to $90 \%$ thickness). Increasing the intramural border zone thickness did not significantly affect the epicardial potential distributions.
\end{abstract}

Keywords:

ST segment, bidomain equation, ischaemia, mathematical model

\section{Introduction}

Cardiac ischaemia occurs when the blood supply to a section of the heart is either reduced of cut off completely, usually the result of a blockage or narrowing of one of the arteries which lie on the surface of the heart. The present numerical study focuses on the electrical consequences of ischaemia during the ST segment of the electrocardiogram (ECG), the phase of the cardiac cycle, which lies between the QRS complex and the $\mathrm{T}$ wave, where the heart is completely depolarised. When cardiac tissue becomes ischaemic, the electrical properties of the affected cells are altered resulting in a change in their transmembrane potential. For a healthy heart, there is very little electrical activity during the ST segment. However, when a section of the heart becomes ischaemic, potential differences between the healthy and ischaemic tissue cause the heart to no longer be in an isoelectric state. This effect can be measured by the extracellular potentials at the heart surface or as a deflection of the ST segment on an ECG trace.

Due to the advancement of computing technology in recent years, numerical studies have become a popular method to study the electrical behaviour of the heart. This is because of their ability to quickly and non-invasively examine scenarios which are either difficult or not possible

\footnotetext{
* Corresponding author

Email address: josef.barnes@griffithuni.edu.au (J.P. Barnes)
}

in an experimental environment. In particular, much research has been dedicated to studying ST segment deviation due to ischaemia $[1,2,3,4]$. From these and other studies, it is well understood that for an ischaemic region which is transmural, ST segment elevation occurs over the ischaemic region. Unfortunately, for the case where the ischaemic region does not extend all the way to the epicardium, the mechanisms which govern this situation are still not yet fully understood [5] and there is no clear consensus on the ST potentials. The variation of results for partial thickness ischaemia is predominately due to the differing choices of vital model parameters such as conductivity and fibre orientation. The earliest of these studies $[6,7]$ were forced to use isotropic conductivities and ignored cardiac fibre rotation due to limited computing power. Work by Johnston et al. [1], however, showed that the anisotropy of cardiac tissue was very important and must be included in order to achieve realistic results. More recent numerical studies [3, 4], using anisotropic conductivity values, have agreed with experimental results [8] for high and low thickness ischaemia. They showed that at low thickness ischaemia, ST depression is centred over the ischaemic region, while at high thicknesses, elevation occurs over the ischaemic region and depression over the ischaemic lateral borders. Differing choices of conductivity values $[2,9]$ between models, however, have resulted in widely varying results for medium thickness ischaemia (30\%-70\%). One consistent result from these studies was the formation of 
'current loops' around the border between ischaemia and healthy tissue, which arise due to the differing transmembrane potentials.

The vast majority of studies on ischaemia during the ST segment have described the ischaemic region using a rectangular prism type geometry. From these studies it can be seen that the presence of 'sharp' edges in the geometry may aid the formation of the current loops. The motivation for the present study is to investigate ischaemic geometries that have fewer of these sharp edges and to study the effect these geometries have on the formation of current loops and extracellular epicardial potentials. Three different ischaemic geometries were used: rectangular, cylindrical and semi-ellipsoidal. The rectangular ischaemic geometry was used for comparison with results by Johnston et al. [1] while the other two geometries represented a decreasing presence of the sharp edges.

\section{Methods}

\subsection{Governing equations}

All the simulations carried out in this study focused on the ST segment (isoelectric phase) of the ECG, meaning that only the steady state bidomain equation was needed to carry out the simulations. This equation has the form

$$
\nabla \cdot\left(M_{i}+M_{e}\right) \nabla \phi_{e}=-\nabla \cdot M_{i} \nabla \phi_{m}
$$

where $M_{i}$ and $M_{e}$ are the intracellular and extracellular conductivity tensors and $\phi_{e}$ and $\phi_{m}$ are the extracellular and transmembrane potentials, respectively. For the heart geometry, a simple slab model was used, based on the model introduced by Johnston et al. [1]. The model consisted of a rectangular slab of ventricular tissue with dimensions $16 \mathrm{~cm} \times 16 \mathrm{~cm} \times 1 \mathrm{~cm}$. The epicardium was situated at $z=0$ and the endocardium at $z=1$. A volume of blood of thickness $20 \mathrm{~cm}$ then sat on top of the tissue. The potential in the blood, $\phi_{b}$, was governed by Laplace's equation

$$
\nabla^{2} \phi_{b}=0
$$

At the outer surface of the model (blood/air and tissue/air interface), insulation conditions were used and at the interface between the blood and tissue, continuity of current and extracellular potential was assumed. Also, the potential at the base of the blood was set to zero, in order to give a unique solution. The boundary conditions for the model can be summed up with the following set of equations:

$$
\begin{array}{ll}
\hat{\mathbf{n}} \cdot \nabla \phi=0 & \text { at the outer surface, } \\
\phi_{e}=\phi_{b}, \quad \sigma_{e}^{t} \frac{\partial \phi_{e}}{\partial z}=\sigma_{b} \frac{\partial \phi_{b}}{\partial z} & \text { at the tissue-blood interface, } \\
\phi_{b}=0 & \text { at the base of the blood. }
\end{array}
$$

where $\hat{\mathbf{n}}$ is the unit vector perpendicular to the outer surface of the model. The conductivity values used in this study were based on the values given by Clerc [10]: $\sigma_{e}^{l}=6.25 \mathrm{mS} / \mathrm{cm}, \sigma_{e}^{t}=2.36 \mathrm{mS} / \mathrm{cm}, \sigma_{i}^{l}=1.74 \mathrm{mS} / \mathrm{cm}$, $\sigma_{i}^{t}=0.193 \mathrm{mS} / \mathrm{cm}$, where the subscripts $i$ and $e$ refer to the intracellular and extracellular spaces, respectively, and the superscripts $l$ and $t$ refer to the directions along and across the fibres, respectively. Linear fibre rotation (anticlockwise direction) was included with a total rotation from the epicardium to endocardium of $120^{\circ}$. The fibres were aligned with the $x$ axis at the epicardium. This was incorporated into the model by modifying the conductivity tensors from Equation (1) in the same way as originally described by Johnston et al. [1]

$$
M_{n}=\left[\begin{array}{ccc}
\left(\sigma_{n}^{l}-\sigma_{n}^{t}\right) c^{2}+\sigma_{n}^{t} & \left(\sigma_{n}^{l}-\sigma_{n}^{t}\right) c s & 0 \\
\left(\sigma_{n}^{l}-\sigma_{n}^{t}\right) c s & \left(\sigma_{n}^{l}-\sigma_{n}^{t}\right) s^{2}+\sigma_{n}^{t} & 0 \\
0 & 0 & \sigma_{n}^{t}
\end{array}\right]
$$

where

$$
c=\cos \left(\frac{2 \pi}{3} z\right), s=\sin \left(\frac{2 \pi}{3} z\right)
$$

and $n=i$ or $e$ for the intracellular or extracellular space. The model was solved using SCIRUN [11], which discretizes the bidomain equation using the finite element method to form the matrix equation

$$
A \Phi=b
$$

and calculates the solution using various iteration methods with preconditioning. A hexahedral mesh was used for all simulations and the nodes were clustered around both the ischaemic border zone and the interface between blood and tissue. As there were three different ischaemic shapes used in this study, this meant the mesh had to be altered to fit the shape of the ischaemic region. In order to test mesh independence, simulations were run using increasingly finer meshes until it was confirmed that the solution converged. It was decided that a mesh with 65 nodes in the $x$ and $y$ direction and 161 nodes in the $z$ direction ( 81 in the tissue and 80 in the blood) was enough to ensure an accurate solution.

\subsection{Ischaemic region}

For the ischaemic region, three different shapes were considered. The first was a rectangular prism with a length of $4 \mathrm{~cm}$ in both the $x$ and $y$ directions. The bottom of the ischaemic region was in contact with the endocardium as shown in figure 1. During the ST segment, it is well known that ischaemic tissue has a lower transmembrane potential than that of healthy tissue. For this model the ischaemic tissue was given a transmembrane potential of $-30 \mathrm{mV}$, while the healthy tissue was given a value of $0 \mathrm{mV}$. Near the border between healthy and ischaemic tissue, the transmembrane potential was varied smoothly from $0 \mathrm{mV}$ to $-30 \mathrm{mV}$ according to the equation

$$
\phi_{m}(x, y, z)=-30 \Psi(x) \Psi(y) \Psi(1-z) .
$$




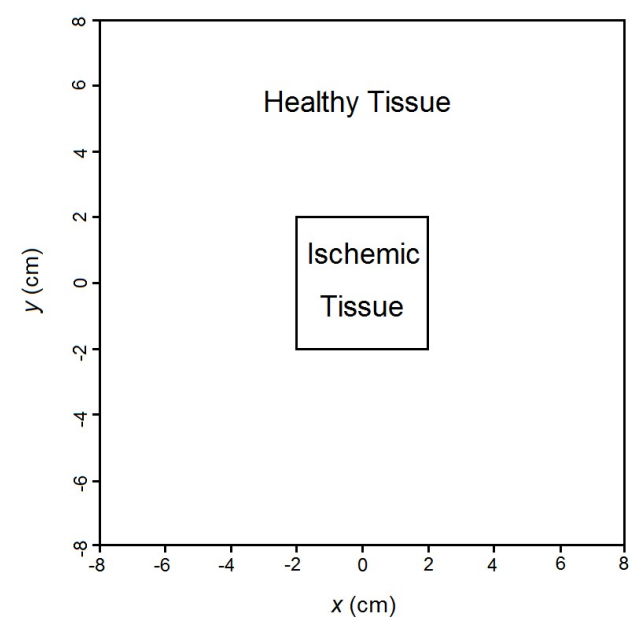

(a)

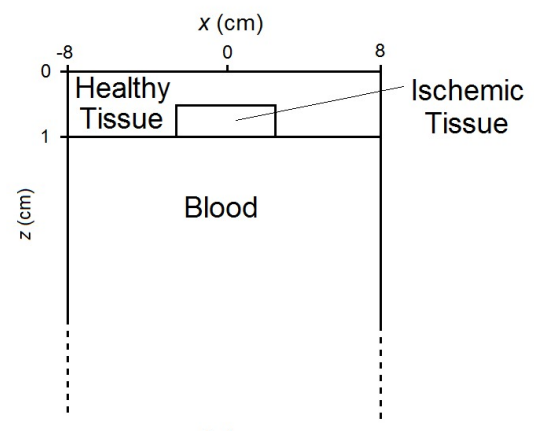

(c)

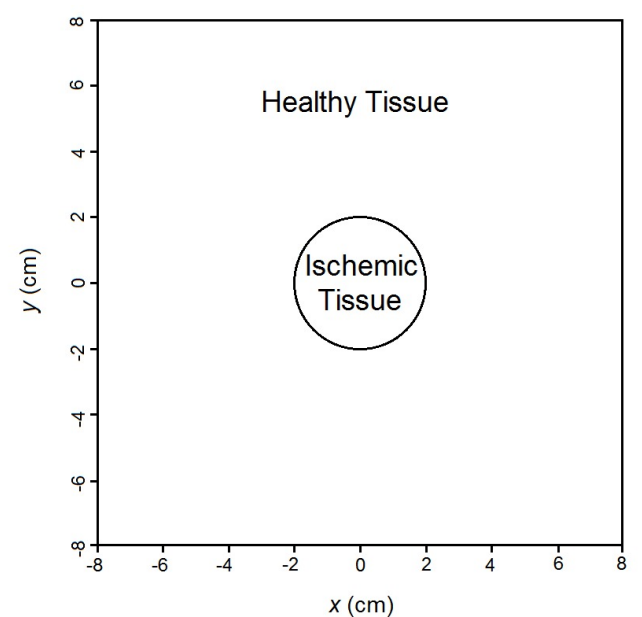

(b)

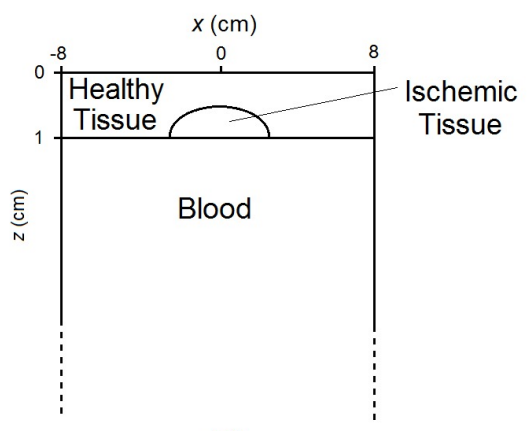

(d)

Figure 1: Slab model used for solving the bidomain equations: (a) cross section along $z$ axis for rectangular ischaemic geometry, (b) cross section along $z$ axis for cylindrical and ellipsoidal ischaemic geometries, (c) cross section along $y$ axis for rectangular and cylindrical ischaemic geometries and (d) cross section along $y$ axis for ellipsoidal ischaemic geometry.

In each spatial direction, the function $\Psi(p)$ is defined as

$$
\Psi(p)= \begin{cases}\frac{1-e^{\frac{-a_{p}}{\lambda_{p}}} \cosh \frac{p}{\lambda_{p}}}{1-e^{\frac{-a_{p}}{\lambda p}}} & |p| \leq a_{p} \\ \frac{e^{\frac{-|p|}{\lambda_{p}}} \sinh \frac{a_{p}}{\lambda_{p}}}{1-e^{\frac{-a_{p}}{\lambda_{p}}}} & |p|>a_{p}\end{cases}
$$

where $a_{p}$ is the half width of the ischaemic region in the $x$ and $y$ directions and the ischaemic thickness in the $z$ direction, while $\lambda_{p}$ is a parameter to adjust the sharpness of the transition. As shown in figure 2 , as the value of $\lambda_{p}$ is increased, the size of the border zone is also increased. For the initial results of this study, a value of 0.01 was chosen for $\lambda_{p}$ in all directions in order to give a sharp border zone.

The second ischaemic geometry considered was a cylindrical shape. It represented a shape in which the edges along the lateral borders were removed and was constructed to have similar size and border zone properties as the rectangular geometry. The cylinder was given a radius of $2 \mathrm{~cm}$ and the equation for the transmembrane potential was

$$
\phi_{m}(r, z)=-30 \Psi(r) \Psi(1-z),
$$

where $r$ is in the radial direction and $\Psi(p)$ is the same as defined in equation (6), except that the parameter $a_{r}$ now represents the radial distance to the centre of the border zone. Again, a value of 0.01 was used for $\lambda_{p}$ in each spatial direction.

The final ischaemic geometry used was an ellipsoidal shape. This shape represented the removal of all edges in the ischaemic region to give a completely smooth border zone. It was constructed by taking the top hemisphere from an oblate spheroid described by the equation

$$
\frac{x^{2}}{a_{r}^{2}}+\frac{y^{2}}{a_{r}^{2}}+\frac{(z-1)^{2}}{a_{z}^{2}}=1
$$

where $a_{r}$ is the radius at $z=1$ and $a_{z}$ is the vertical, conjugate radius. For consistency with the previous ischaemic geometries, a value of $2 \mathrm{~cm}$ was chosen for $a_{r}$. The transmembrane potential was governed by the equation

$$
\phi_{m}(d)=-30 \Phi(d),
$$

where the function $\Phi(d)$ is defined as

$$
\Phi(d)= \begin{cases}\frac{1}{2} e^{-\frac{d}{\lambda}} & \text { outside ischaemic region } \\ 1-\frac{1}{2} e^{-\frac{d}{\lambda}} & \text { inside ischaemic region }\end{cases}
$$




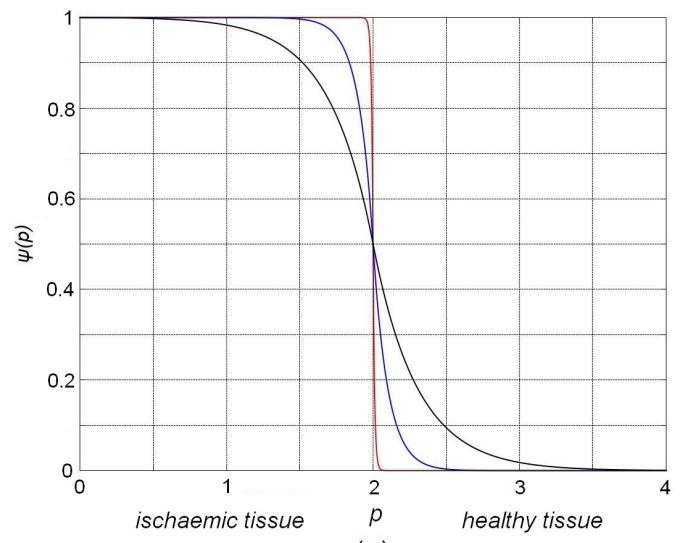

(a)

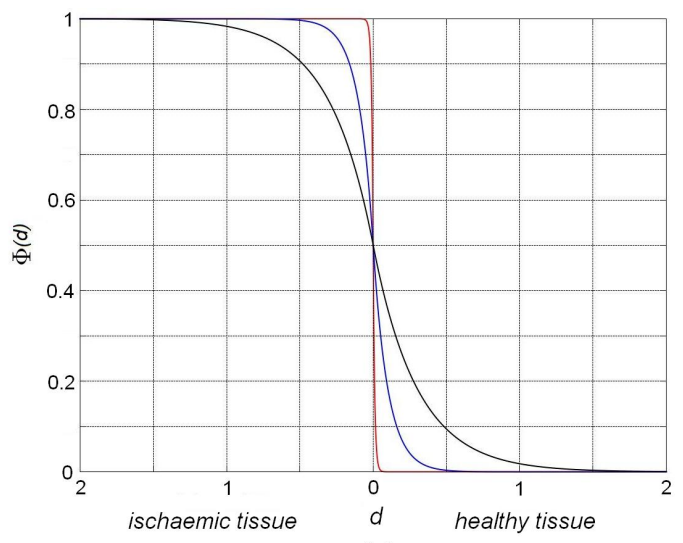

(b)

Figure 2: Comparison of the $\Psi(p)$ (a) and $\Phi(d)$ (b) functions for $\lambda=0.01$ (red), $\lambda=0.1$ (blue) and $\lambda=0.5$ (black).

and $d$ is the perpendicular distance to the centre of the border zone. The $\lambda$ parameter again a controls the sharpness of the transition. Figure 2 shows that the difference in the width of the border zone is negligible for $\Psi(p)$ and $\Phi(d)$, using equivalent values of $\lambda$.

\section{Results and discussion}

\subsection{Unmodified slab model}

The results for the unmodified slab model show that, at relatively small thicknesses of ischaemia $\left(a_{z}<30 \%\right)$, the epicardial potential distribution (EPD) has a single depression located directly over the ischaemic region. This depression then begins to separate into three depressions as ischaemic thickness is increased, one remaining over the region of ischaemia and two over opposing lateral borders. By the time the thickness reaches $50 \%$, the three depressions become quite distinct, as can be seen in figure 3 (top row). Once the ischaemic thickness exceeds $70 \%$, two small areas of elevation appear over opposing corners of the ischaemic region. These areas of elevation quickly spread and cover the area above the ischaemic region, leaving one elevation and two depressions at thicknesses above $90 \%$. This behaviour can be understood better by studying the current paths inside the tissue. What these show, for the unmodified slab model (figure 4, top row), is that, at low levels of ischaemia, current loops are formed around the lateral borders of the ischaemic region. As the ischaemic thickness is increased, separate loops form around the top edges which rotate in the opposite direction to the more dominant loop around the bottom edges. This is where the single depression splits into three depressions. Once the ischaemic thickness is too large, the effects of the top loop start to dominate and this is when positive potentials at the epicardium occur.

The EPDs obtained using a cylindrical shaped ischaemic geometry (figure 3, middle row) are quite similar to those for the rectangular geometry. A single depression at low levels of ischaemia separates into three depressions as the thickness increased to approximately 50\%. At higher ischaemic thicknesses, very similar to the rectangular geometry, elevation begins to appear above the lateral border and quickly cover the area above the ischaemic region. The current paths for the cylindrical ischaemic model are also very similar to the rectangular model, with the formation of current loops around the top and bottom sharp edges of the ischaemic zone. These results suggest that the presence of sharp edges on the lateral borders may not play a significant role in the determining epicardial potentials.

When comparing the epicardial distributions for the ellipsoidal ischaemic model to the previous two, differences immediately become apparent. At low levels of ischaemia, the results agree well with the previous models, with a single depression centred over the ischaemic region. It is at higher thicknesses where the differences are noticed. As ischaemic thickness is increased beyond 30\%, the single depression separates into only two depressions, which move outward until they are over the lateral edge of the ischaemic region. As the ischaemic thickness is increased above $60 \%$, elevation begins to occur over the centre of the ischaemic region and move outwards. This differs from the previous two situations where elevation started at the lateral borders and moved inward. This means that the area of maximum elevation is over the centre of the ischaemic region instead of above the lateral borders.

Differences can also be seen in the current paths of the ellipsoidal ischaemic model (figure 4, bottom row). For low levels of ischaemia, the current paths agree with the previous models, showing only the primary loop around the edges of the ischaemic region. As the ischaemic thickness increases and the top surface becomes increasingly curved, a current sink begins to form at the base edges. This causes the current to move outward from the centre and therefore raises the potential over the ischaemic region, with the result that the central depression, and also the elevation found at high ischaemic thicknesses, both disappear. 


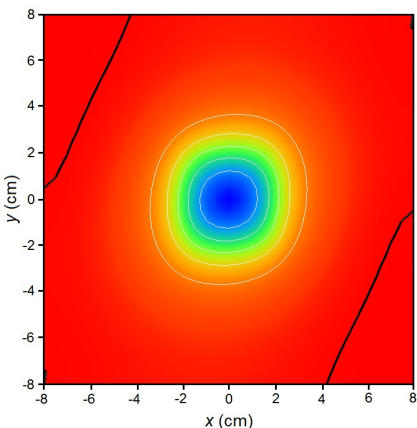

(a)

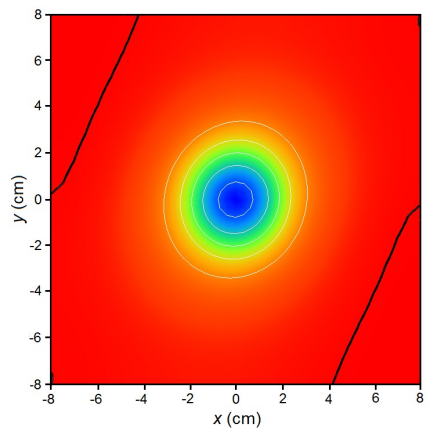

(e)

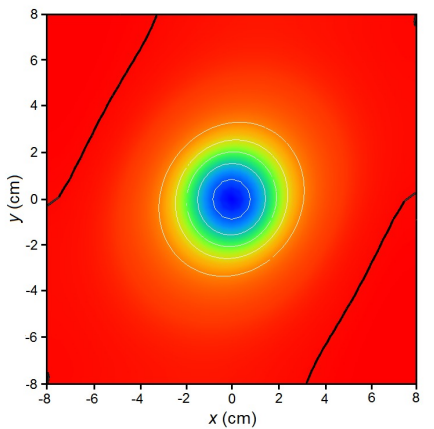

(i)

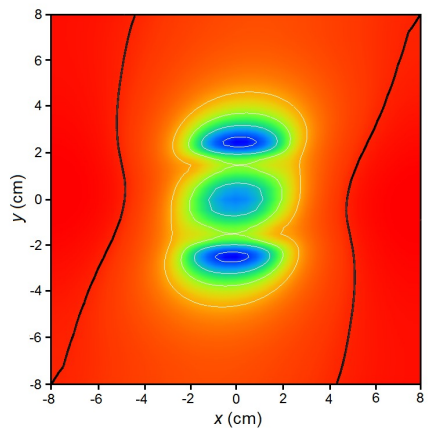

(b)

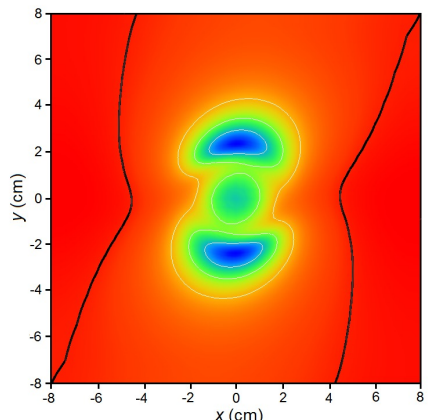

(f)

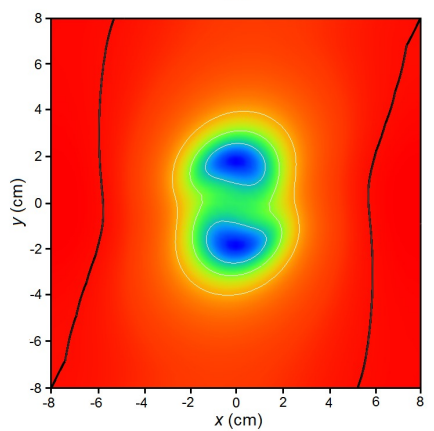

(j)

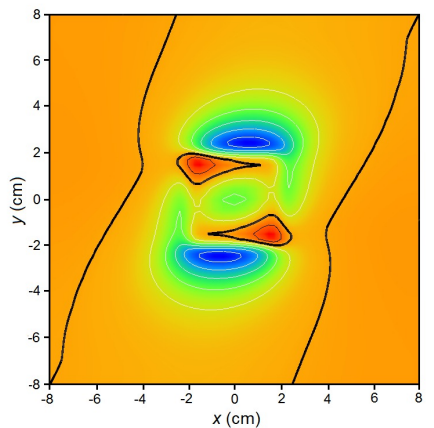

(c)

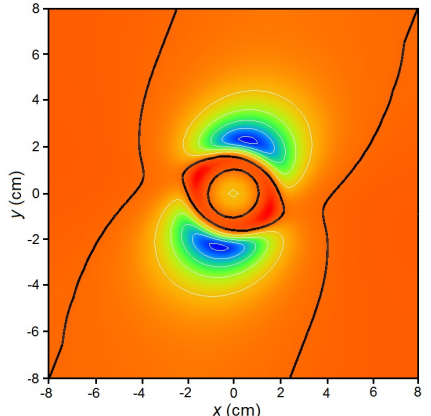

(g)

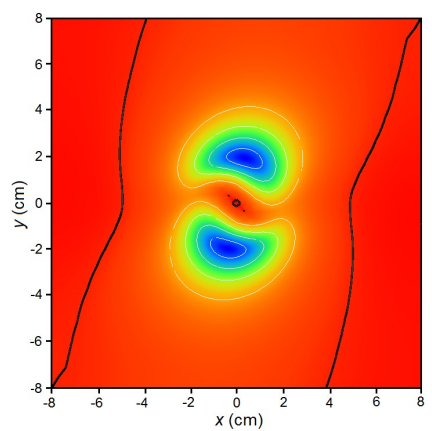

(k)

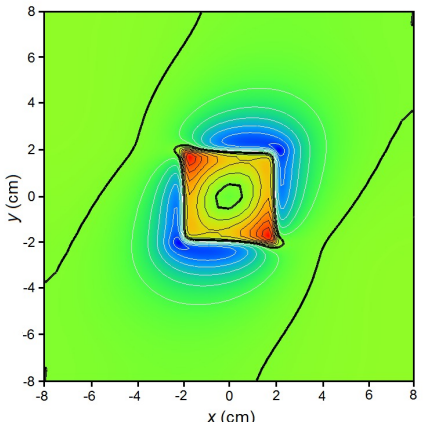

(d)

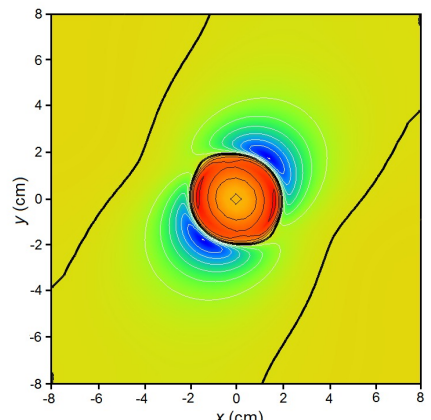

(h)

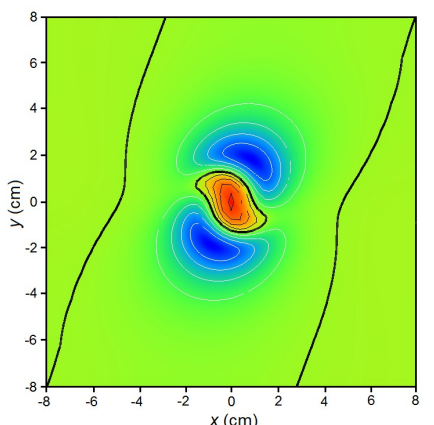

(I)

Figure 3: Extracellular EPDs for the initial results: (a), (d), (g), (j) rectangular ischaemic geometry for $10 \%, 50 \%, 70 \%, 90 \%$ ischaemia, respectively; (b), (e), (h), (k) cylindrical ischaemic geometry for 10\%, 50\%, 70\%, 90\% ischaemia, respectively and (c), (f), (i), (l) ellipsoidal ischaemic geometry for $10 \%, 50 \%, 70 \%, 90 \%$ ischaemia, respectively.

\subsection{Changing the Ischaemic Conductivity}

The results in the previous section assumed that the conductivity inside the ischaemic region is the same as that in healthy tissue. It is accepted that there are three phases of conductivity change during ischaemia:

- an immediate sharp decrease in longitudinal extracellular conductivity at the onset of ischaemia $[12,13]$;

- a few minutes later, a gradual decrease in longitudinal extracellular conductivity occurs and this lasts for at least 15 minutes [14], and

- a sharp decrease in longitudinal intracellular conductivity, which occurs 15-30 minutes after the onset of ischaemia $[13,15]$.

Several mechanisms have been proposed for the three phases of ischaemia. Kiéber and Riegger [12] proposed that the initial sharp decrease of longitudinal extracellular conductivity was due to the collapse of capillaries. Results from Fleischhauer et al. [16] and Stinstra et al. [17], however, suggested that a change in capillary volume would not affect the extracellular conductivity significantly. Stinstra et al. proposed an alternative mechanism, in which a rapid decrease in the size of the interstitial space, due to fluid flow, is responsible for the first phase. The mechanism for the second phases is somewhat less controversial and predominately occurs due to cell swelling [13]. Likewise the third phase is accepted to occur due to the closing of gap junctions $[13,18]$.

In order to account for these conductivity changes, the model for this study was altered by decreasing the conductivity in the ischaemic region according to the results of Stinstra et al. [17] and Kiéber and Riegger [12], including all three phases of ischaemia. This involved decreasing the 


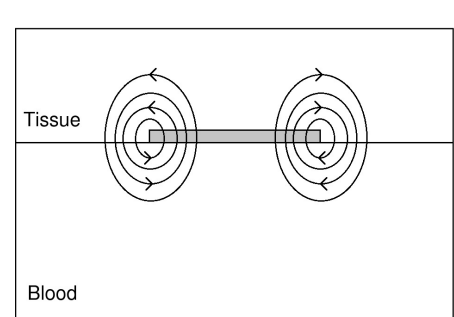

(a)

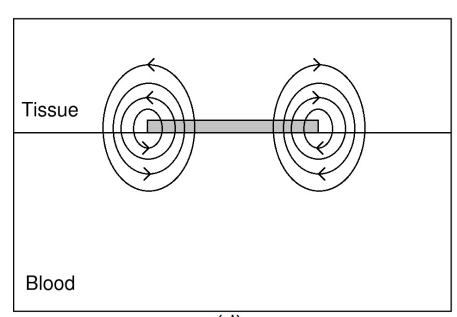

(d)

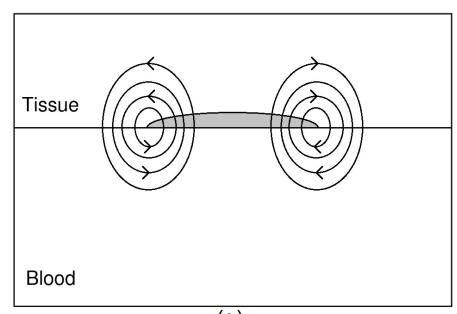

(g)

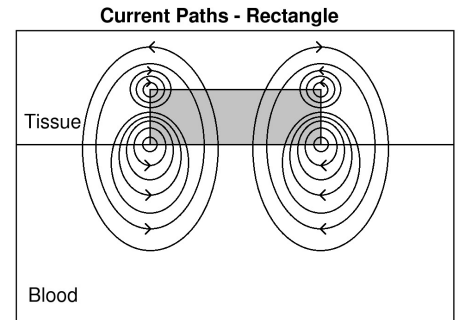

(b)

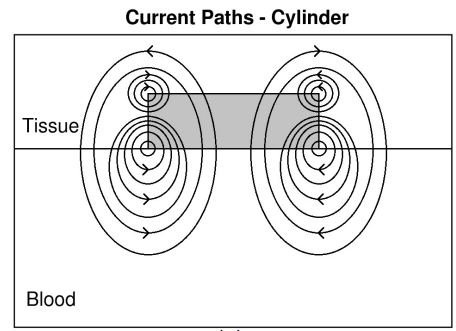

(e)

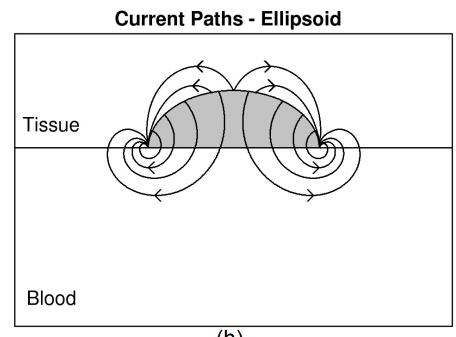

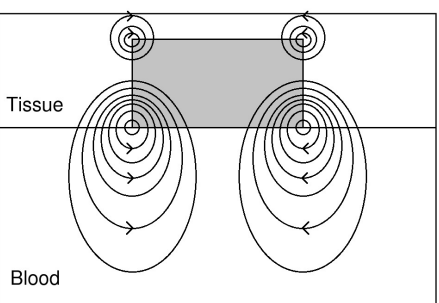

(c)

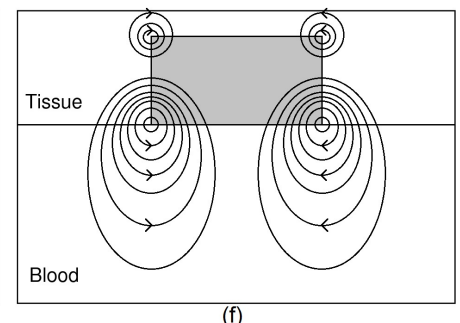

(f)

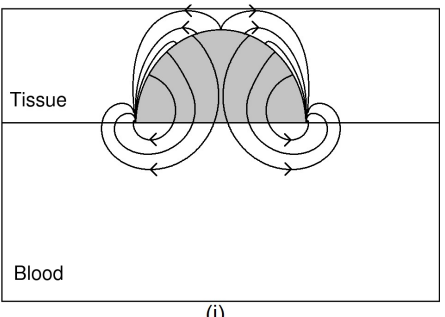

Figure 4: Current paths for the initial results; (a), (b), (c) rectangular ischaemic geometry for low, medium and high ischaemic thickness, respectively; (d), (e), (f) cylindrical ischaemic geometry for low, medium and high ischaemic thickness, respectively and (g), (h), (i) ellipsoidal ischaemic geometry for low, medium and high ischaemic thickness, respectively.

\begin{tabular}{|l|l|l|l|l|}
\hline & $\sigma_{e}^{l}$ & $\sigma_{e}^{t}$ & $\sigma_{i}^{l}$ & $\sigma_{i}^{t}$ \\
\hline Healthy Tissue & 0.625 & 0.236 & 0.174 & 0.019 \\
\hline Ischaemic Tissue & 0.400 & 0.236 & 0.017 & 0.019 \\
\hline
\end{tabular}

Table 1: Conductivity values for healthy and ischaemic tissue. All values have units of $\mathrm{S} / \mathrm{m}$.

extracellular longitudinal conductivity by approximately $35 \%$ and the intracellular longitudinal conductivity by approximately $90 \%$. The transverse conductivities were not altered as they have shown to not significantly change during ischaemia [17, 12]. Table 1 summarises the changes in conductivity values. As with the transmembrane potential, a smooth transition between conductivity values was included to represent the border zone between healthy and ischaemic tissue.

Comparing the EPDs of the rectangular ischaemic geometry with these changed conductivity values (figure 5, top row) to the initial results, noticeable differences can be seen at medium and high thicknesses of ischaemia. For low thicknesses $\left(a_{z}<30 \%\right)$, the distributions look quite similar, with only one central depression and comparable minimum potentials. As the degree of ischaemia is increased beyond $30 \%$, the central depression does not immediately separate into the three depressions, as seen previously. The two secondary depressions begin to appear only after the ischaemic thickness reaches $70 \%$. Also unlike the previous results, the point of maximum depression now resides in the central depression, rather than the secondary ones. It is not until the ischaemic thickness exceeds $80 \%$ that elevation occurs on the epicardium. This happens over two diagonal corners of the ischaemic region. Even at $90 \%$ ischaemia, there is still a large area of depression over the ischaemic region. The elevation only starts to spread rapidly once the ischaemic region is increased almost to $100 \%$, approximately when the border zone starts to show on the epicardium.

The model with a cylindrical ischaemic geometry (figure 5 , middle row) shows a very similar pattern to its rectangular counterpart. At low degrees of ischaemia, there is little difference from the unaltered conductivity case. But where the unaltered case starts to separate into three depressions, the same does not happen with the altered model. One key difference between the cylindrical and rectangular models is that the central depression in the cylindrical is not as deep as in the rectangular model at large degrees of ischaemia.

The differences in the ellipsoidal ischaemic model (figure 5 , bottom row) also follow similar patterns in that the sep- 


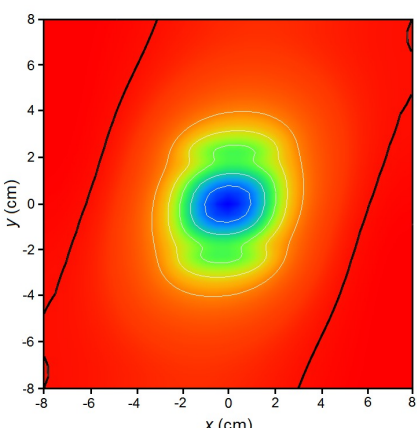

(a)

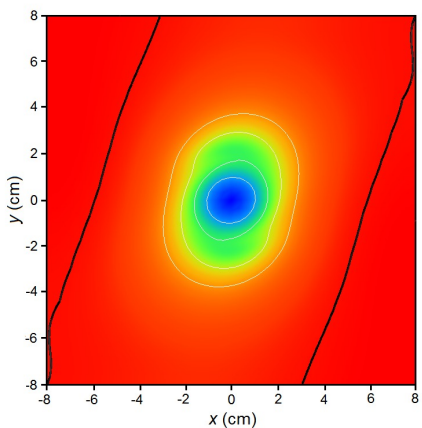

(d)

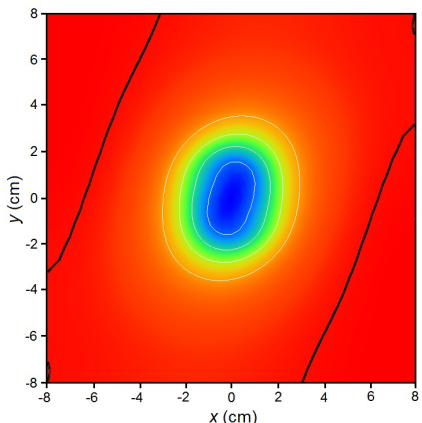

(g)

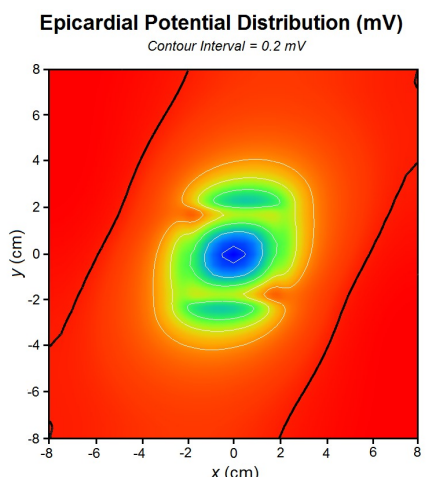

(b)

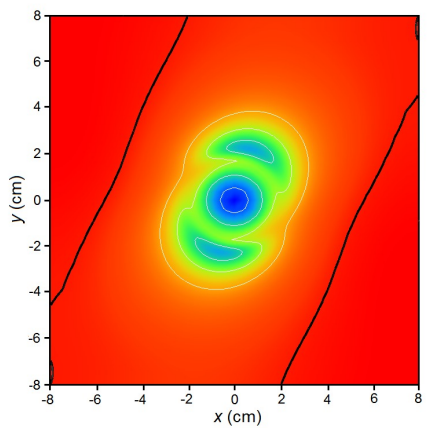

(e)

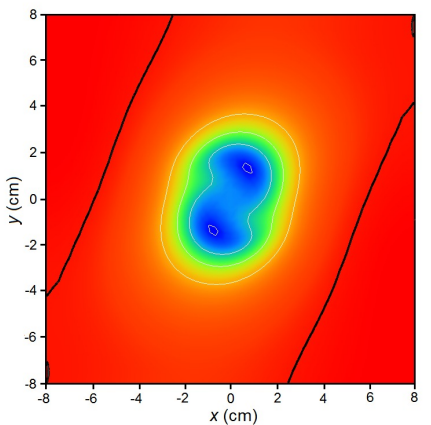

(h)

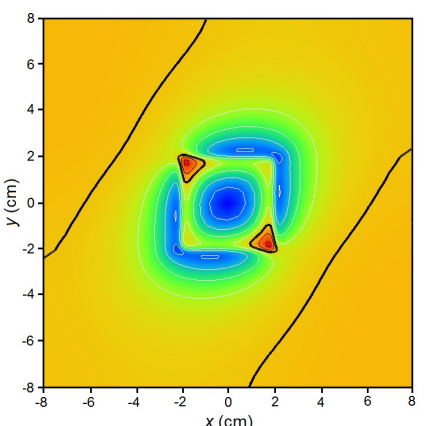

(c)

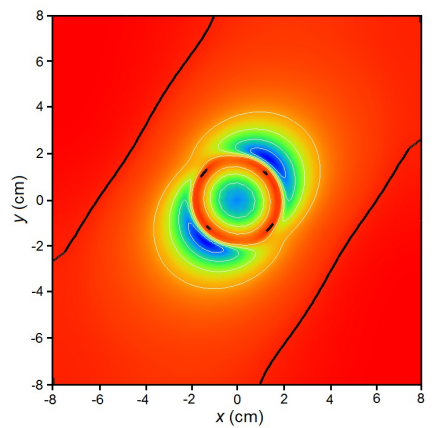

(f)

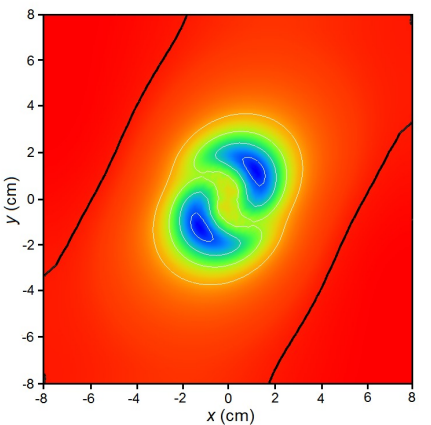

(i)

Figure 5: Extracellular EPDs for the reduced conductivity results: (a), (d), (g) rectangular ischaemic geometry for 50\%, 70\%, 90\% ischaemia, respectively; (b), (e), (h) cylindrical ischaemic geometry for 50\%, 70\%, 90\% ischaemia, respectively and (c), (f), (i) ellipsoidal ischaemic geometry for $50 \%, 70 \%, 90 \%$ ischaemia, respectively.

aration of the central depression occurs at higher degrees of ischaemia than in the unaltered conductivity model. Elevation over the ischaemic region also does not start to appear until the ischaemic thickness is at least 95\%. An interesting feature of the ellipsoidal model is that, once the ischaemic region extended to the epicardium, the area of elevation over the ischaemic region was much smaller than the unaltered conductivity case, making it more difficult to detect in clinical situations.

The current paths for all ischaemic models also change significantly when the ischaemic conductivity is reduced. As with the epicardial potentials, the current paths for the cylindrical and rectangular models show a slower response to the increase in ischaemic thickness. For low levels of ischaemia, there is again the formation of two current loops around the lateral borders. As the ischaemic thickness increases, however, the separate loops on the top edges does not form until much later than with the previous results. This aids in understanding why the separation of the three depressions does not become apparent until quite large levels of ischaemia. These loops do not begin to dominate at the epicardium until the ischaemia is near full thickness, which is why elevation does not occur until this point.

The ellipsoidal model also shows similar patterns to the other models, with changes in the current loops not occurring until much higher levels of ischaemia. The current sink found in the initial results is not as strong for the reduced conductivity situation, and the current loops start to form a spiral pattern. This means that the magnitude of the outward current over the centre of the ischaemic region is reduced and hence, the rise in the central potentials does not occur. Similar to the rectangular and cylindrical models, it is not until the ischaemia becomes transmural 


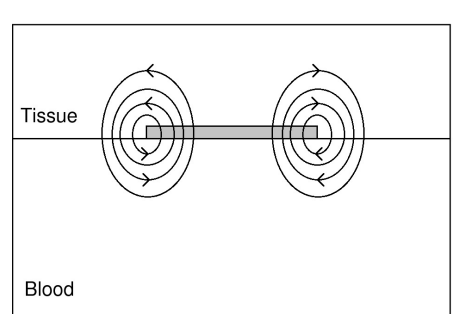

(a)

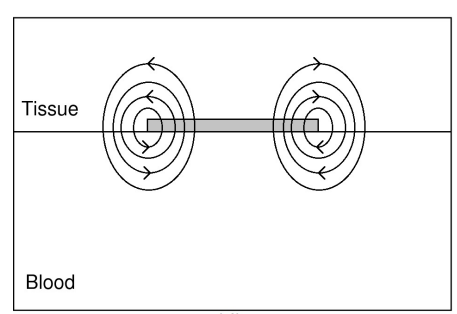

(d)

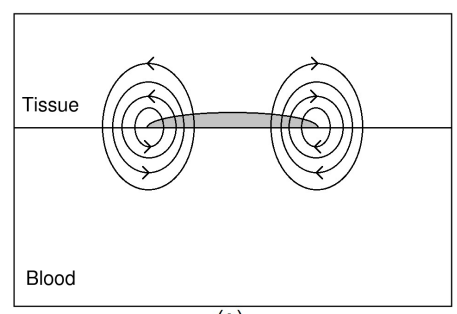

(g)

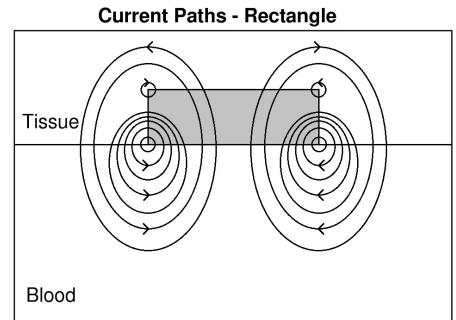

(b)

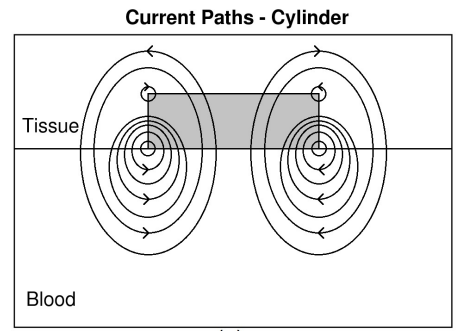

(e)

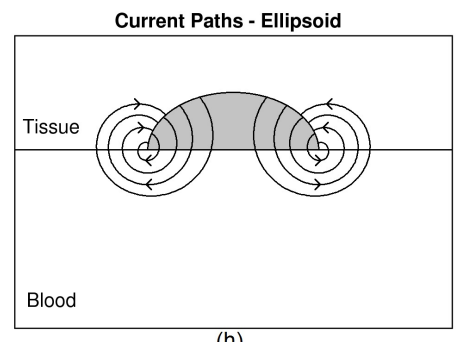

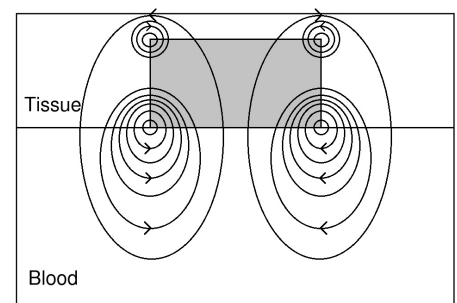

(c)

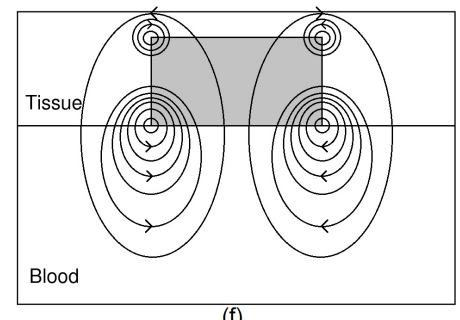

(f)

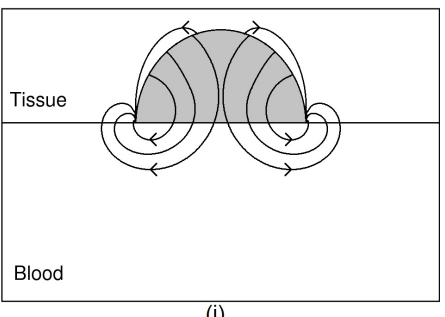

Figure 6: Current paths for the reduced conductivity results: (a), (b), (c) rectangular ischaemic geometry for low, medium and high ischaemic thickness, respectively; (d), (e), (f) cylindrical ischaemic geometry for low, medium and high ischaemic thickness, respectively and (g), (h), (i) Ellipsoidal ischaemic geometry for low, medium and high ischaemic thickness, respectively.

that the current sinks at the bottom edges of the ischaemic region affect potentials at the epicardium.

\subsection{Changing the Ischaemic Border zone}

Another simplistic assumption in the original slab model was the size of the ischaemic border zone. The original model used a $\lambda$ value of 0.01 in the transmembrane potential equation which resulted in a border zone which was less than 1mm thick. Experimental studies [19, 20] have shown that the border zone in the intramural direction is much larger than this, varying from 8 to $15 \mathrm{~mm}$. The slab model was corrected to account for these larger border zones by increasing the $\lambda_{x}$ and $\lambda_{y}$ values to 0.5 , which resulted in a border zone in the $x$ and $y$ directions of approximately $1 \mathrm{~cm}$, as shown in figure 2 . Simulations were also run, using a $\lambda$ value of 0.1 in the $x$ and $y$ directions, to look at the transition between the sharp and thick border zone scenarios.

The value of $\lambda$ in the $z$ direction was necessarily left at 0.01 in order to satisfy the boundary conditions of the computational model. The insulation condition on the model meant that the gradient of the transmembrane potential needed to tend to zero at the epicardium, therefore limiting the value of $\lambda_{z}$ which could be used. For the ellipsoidal ischaemic geometry, a different method was required to achieve similar border zone conditions. Since there are no distinct lateral and transverse borders, it was decided to vary the value of $\lambda$ linearly with $z$ so that it was 0.5 (or 0.1 in the intermediate scenario) at the endocardium and 0.01 at the top of the ischaemic zone.

The reduced conductivity values were used in all the simulations with varying border zone thicknesses.

The results obtained from varying the border zones in the $x$ and $y$ direction are quite similar to the case with sharp border zones. For the case where $\lambda=0.1$ for the intramural borders, the EPDs are almost identical to the reduced conductivity results. The only noticeable differences are slight changes in the minimum and maximum potentials.

When the intramural border zone width increases to approximately $1 \mathrm{~cm}$, the differences are much more noticeable. However, the distributions are still quite similar to the sharp border scenario. For lower ischaemic thicknesses $(<30 \%)$, the EPDs look the same as with all previous results, with a single depression located over the centre of the ischaemic region. The major changes come from higher ischaemic thicknesses where, in the case of the rectangular and cylindrical ischaemic geometries, a noticeable softening of the two depressions sitting over the border zone can be seen. Interestingly, this does not affect the central 


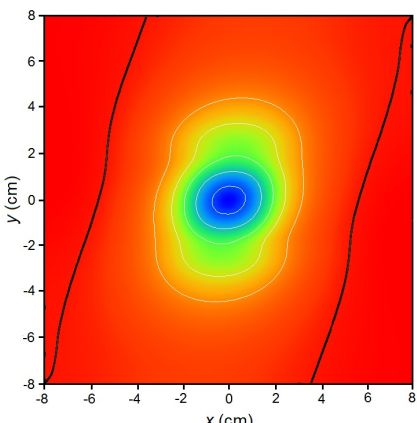

(a)

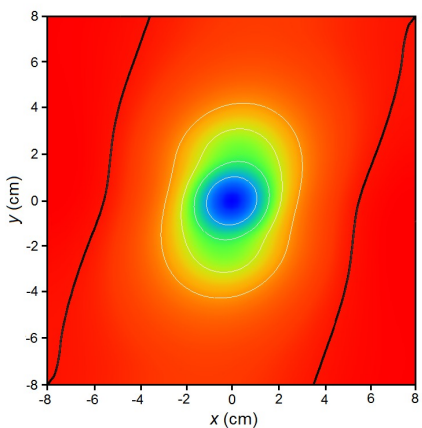

(d)

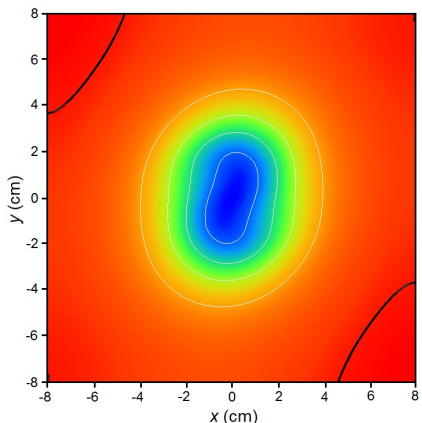

(g)

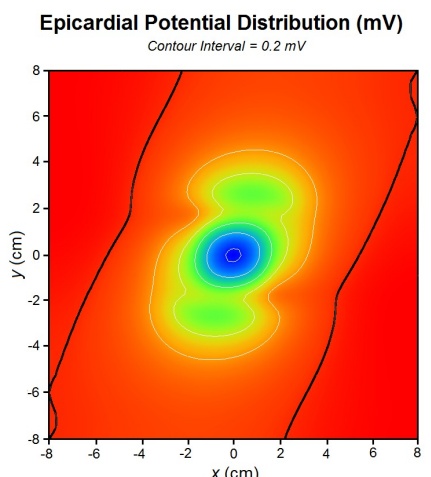

(b)

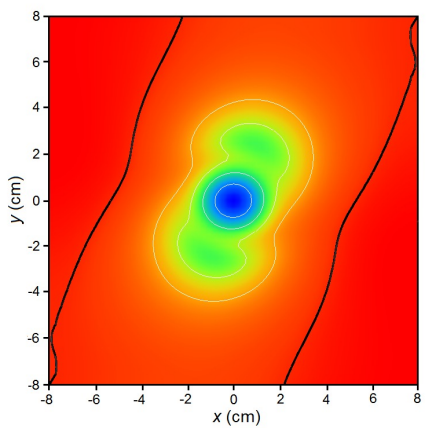

(e)

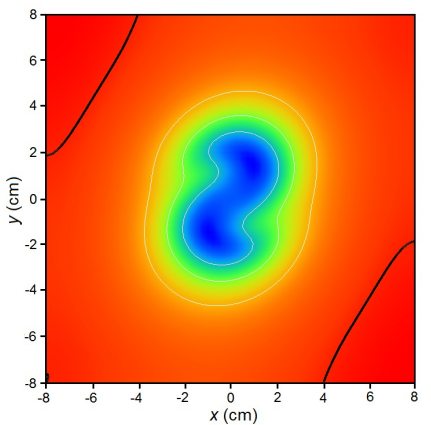

(h)

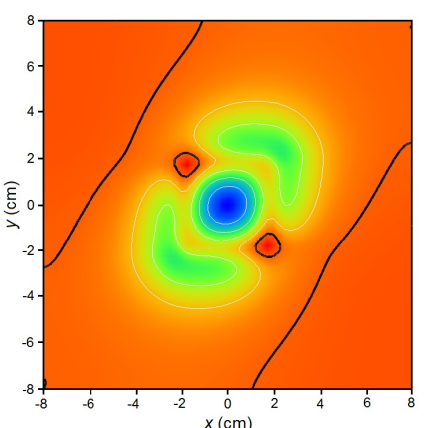

(c)

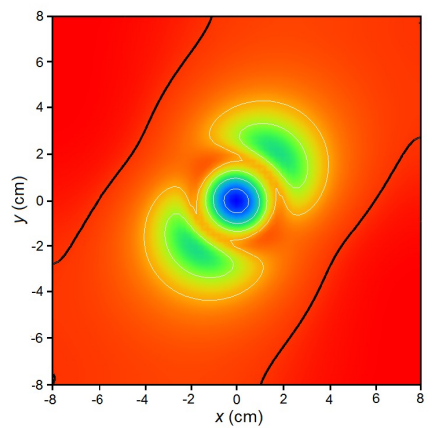

(f)

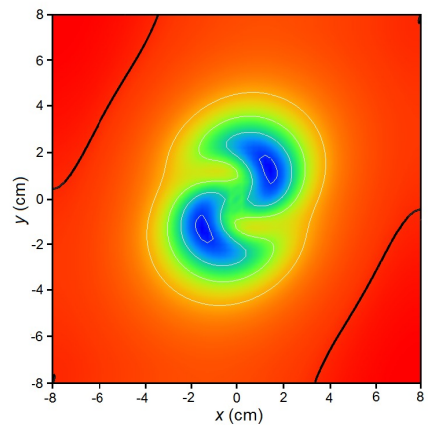

(i)

Figure 7: Extracellular EPDs for the increased border zone results: (a), (d), (g) rectangular ischaemic geometry for $50 \%, 70 \%, 90 \%$ ischaemia, respectively; (b), (e), (h) cylindrical ischaemic geometry for 50\%, 70\%, 90\% ischaemia, respectively and (c), (f), (i) ellipsoidal ischaemic geometry for $50 \%, 70 \%, 90 \%$ ischaemia, respectively.

depression, which still has very similar potentials to the sharp border scenario for all levels of ischaemia. The ellipsoidal geometry, however, does not have this same feature, with the potentials at the centre of the two depressions very similar to previous results.

One consistent difference with all ischaemic geometries is the prolonged absence of elevation, even at very high levels of ischaemia. At $90 \%$ ischaemia, only the rectangular ischaemic model shows any elevation. Although the cylindrical model doe not show elevation at this stage, the potentials over the border zone are very close to zero and elevation does occur if the ischaemic thickness increases by a further $1-2 \%$. So the rectangular and cylindrical models were still showing quite similar behaviour. On the other hand, the ellipsoidal ischaemic model (figure 7, bottom row), like the sharp border scenario, does not show any signs of elevation occurring until the level of ischaemia is at least (95\%).

The current paths for this scenario are also very similar to the previous results. The only noticeable difference which can be seen in any of the ischaemic models is a decrease in magnitude of the current through the lateral ischaemic borders. Since the transmembrane potential gradients in this scenario are lower than with previous results (due to the wider borders) this is of course expected and not surprising.

\section{Conclusions}

The work presented here illustrates the difference, in extracellular EPDs during the ST segment, between scenarios of increasing complexity and realism. The change in behaviour found from using different geometries for the 
ischaemic region are quite significant and results in very different patterns in the EPDs.

Another important finding is that the conductivity inside the ischaemic region cannot safely be assumed to be the same as that of healthy tissue. The reduction of conductivity values for ischaemic tissue results in drastic changes in the EPDs, especially for higher level ischaemia.

The thickness of the transmural borders, however, has comparatively little effective on the EPDs, with results quite similar to the case with sharp borders. Although differences can be noticed when the border zone width is within experimentally observed values, they are insignificant when compared to the results found by changing the shape and conductivity of the ischaemic region.

\section{Acknowledgements}

This work was made possible in part by software from the NIH/NIGMS Center for Integrative Biomedical Computing, 2P41 RR0112553-12.

\section{References}

[1] P. R. Johnston, D. Kilpatrick, C. Y. Li, The Importance of Anisotropy in Modeling ST Segment Shift in Subendocardial Ischaemia, IEEE Trans Biomed Eng 48 (12) (2001) 1366-1376.

[2] P. R. Johnston, D. Kilpatrick, The Effect of Conductivity Values on ST Segment Shift in Subendocardial Ischaemia, IEEE Trans Biomed Eng 50 (2) (2003) 150-158. doi:10.1109/TBME.2002.807660.

[3] R. S. MacLeod, S. Shome, J. Stinstra, B. B. Punske, B. Hopenfeld, Mechanisms of Ischemia-Induced ST-Segment Changes, J Electrocardiol 38 (4, Suppl. S) (2005) 8-13. doi:10.1016/j.jelectrocard.2005.06.095.

[4] M. Potse, R. Coronel, S. Falcao, A. LeBlanc, A. Vinet, The Effect of Lesion Size and Tissue Remodeling on ST Deviation in Partial-Thickness Ischemia, Heart Rhythm 4 (2) (2007) 200206. doi:10.1016/j.hrthm.2006.10.022.

[5] B. Rodriguez, N. Trayanova, D. Noble, Modeling Cardiac Ischemia, Ann NY Acad Sci 1080 (2006) 395-414.

[6] R. P. Holland, H. Brooks, Spatial and Non-Spatial Influences on TQ-ST Segment Deflection of Ischemia - Theoretical and Experimental Analysis in Pig, J Clin Invest 60 (1) (1977) 197214.

[7] G. T. Smith, G. G. Geary, W. Blanchard, T. H. Roelofs, W. Ruf, J. J. Mcnamara, An Electrocardiographic Model of Myocardial Ischemic Injury, J Electrocardiol 16 (3) (1983) 223-233.

[8] D. S. Li, C. Y. Li, A. C. Yong, D. Kilpatrick, Source of Electrocardiographic ST Changes in Subendocardial Ischemia, Circ Res 82 (9) (1998) 957-970.

[9] B. Hopenfeld, J. G. Stinstra, R. S. MacLeod, The Effect of Conductivity on ST-Segment Epicardial Potentials Arising from Subendocardial Ischemia, Ann Biomed Eng 33 (6) (2005) 751763. doi:10.1007/s10439-005-3236-2.

[10] L. Clerc, Directional Differences of Impulse Spread in Trabecular Muscle from Mammalian Heart, Journal of Physiology 255 (2) (1976) 335-346.

[11] SCIRun: A Scientific Computing Problem Solving Environment, Scientific Computing and Imaging Institute (SCI). [link]. URL http://www.scirun.org

[12] A. G. Kleber, C. B. Riegger, M. J. Janse, Extracellular K+ and $\mathrm{H}+$ Shifts in Early Ischemia: Mechanisms and Relation to Changes in Impulse Propagation, Journal of Molecular and Cellular Cardiology 19 (Suppl. 5) (1987) 35-44.
[13] W. Smith, W. Fleet, T. Johnson, C. Engle, W. Cascio, The ib phase of ventricular arrhythmias in ischemic in-situ porcine heart is related to changes in cell-to-cell electrical coupling, Circulation 92 (10) (1995) 3051-3060.

[14] G. Yan, J. Chen, K. Yamada, A. Kleber, P. Corr, Contribution of shrinkage of extracellular space to extracellular $\mathrm{K}+$ accumulation in myocardial ischaemia of the rabbit, Journal Of Physiology-London 490 (1) (1996) 215-228.

[15] S. Jain, R. Schuessler, J. Saffitz, Mechanisms of delayed electrical uncoupling induced by ischemic preconditioning, Circulation Research 92 (10) (2003) 1138-1144. doi:10.1161/01.RES.0000074883.66422.C5.

[16] J. Fleischhauer, L. Lehmann, A. Kleber, Electrical resistances of interstitial and microvascular space as determinants of the extracellular electrical-field and velocity of propagation in ventricular myocardium, Circulation 92 (3) (1995) 587-594.

[17] J. G. Stinstra, S. Shome, B. Hopenfeld, R. S. MacLeod, Modelling passive cardiac conductivity during ischaemia, Medical \& Biological Engineering \& Computing 43 (6) (2005) 776-782.

[18] L. Owens, T. Fralix, E. Murphy, W. Cascio, L. Gettes, Correlation of ischemia-induced extracellular and intracellular ion changes to cell-to-cell electrical uncoupling in isolated bloodperfused rabbit hearts, Circulation 94 (1) (1996) 10-13.

[19] M. Janse, J. Cinca, H. Morena, J. Fiolet, A. Kleber, G. Devries, A. Becker, D. Durrer, Border zone in myocardial ischemia electro-physiological, metabolic, and histochemical correlation in the pig-heart, Circulation Research 44 (4) (1979) 576-588.

[20] R. Coronel, J. Fiolet, F. Wilmsschopman, A. Schaapherder, T. Johnson, L. Gettes, M. Janse, Distribution of extracellular potassium and its relation to electrophysiologic changes during acute myocardial ischemia in the isolated perfused porcine heart, Circulation Research 77 (5) (1988) 1125-1138. 